\title{
Patterns of reinnervation and motor unit recruitment in human hand muscles after complete ulnar and median nerve section and resuture
}

\author{
CHRISTINE K THOMAS, RICHARD B STEIN, TESSA GORDON,* \\ ROBERT G LEE, $\$ M$ GEORGE ELLEKER†
}

From the Departments of Physiology, Pharmacology* and Medicine, $\dagger$ University of Alberta, Edmonton, and Department of Clinical Neurosciences, $\ddagger$ University of Calgary, Calgary, Canada

SUMMARY Following complete ulnar or above-elbow median nerve sections, there was no significant correlation between motor unit size (twitch amplitude) and recruitment threshold, as assessed by spike triggered averaging. This absence of orderly recruitment was attributed to misdirection of motor axons during regeneration. Following median nerve section at wrist level, where the reinnervated muscles have more synergistic actions, orderly recruitment by size appeared to be re-established. Thus, the size principle of motor unit recruitment can be re-established after nerve section in humans, if motor axons innervate their original muscles or ones with closely synergistic functions.

If we want to tie a shoelace, write a letter, eat, or play a musical instrument, we are confronted with the need to control our hand muscles precisely. Our ability to co-ordinate these and many other fine movements is generally assumed to result from direct corticospinal connections to the motoneurons innervating the hand muscles $^{12}$ and from an orderly recruitment of motoneurons from small to large within each spinal motor pool. ${ }^{34}$

One notable exception to this recruitment pattern occurred after resuture of a completely severed ulnar nerve in the forearm. Normal motor unit twitch amplitudes were re-established but the threshold force for recruitment of these reinnervated motor units remained abnormal. In addition to a disordered recruitment of motor units, these individuals showed poor motor co-ordination for fine movements. ${ }^{5}$ In these patients, the axons had been severed and so disconnected from a pathway to their peripheral end organs. As the ulnar nerve contains axons which innervate several intrinsic hand muscles, many muscles were denervated. During regeneration, the axons had to grow across the suture line and along the endoneurial tubes to innervate the denervated muscle fibres

Address for reprint requests: Dr RB Stein, Department of Physiology, University of Alberta. Edmonton. Alberta. Canada. T6G 2H7.

Received 22 April 1986. Accepted 25 May 1986. in the periphery. ${ }^{6}$ These axons had opportunities to reinnervate their original or foreign muscles. Therefore, the disordered recruitment of reinnervated motor units could have resulted from misdirection of motor axons to foreign muscles with different functions and/or from changes in the activity of the motoneurons.

Studies on animals have shown that severed motor axons do not reinnervate their original muscles specifically. ${ }^{7}$ Motor axons also reinnervate muscle fibres irrespective of their original type. This is reflected by the altered distribution of the muscle fibres of reinnervated muscle units. They tend to be grouped together, rather that scattered throughout the muscle in the normal mosaic pattern. ${ }^{89}$ As most animal muscles are heterogeneous, ${ }^{10}$ these newly formed muscle units must contain fibres with different histochemical composition and contractile properties. However, reinnervated motor units become like normal motor units with time, in that their muscle fibres show homogeneous histochemical composition ${ }^{11}$ and normal contractile properties. ${ }^{12}$ These changes must have involved transformation of many muscle fibres.

Similarly, regenerating human motor axons are unlikely to grow along their original endoneurial tubes, although the pattern of motor innervation in reinnervated human muscles has not been demonstrated. If reinnervation is specific, a change in the activity of the motoneurons could alter the threshold 
forces for voluntary recruitment of the motor units and thereby produce disorderly recruitment within the reinnervated muscles. Alternatively, non-specific reinnervation of muscles could produce disorderly motor unit recruitment because of misdirection of motor axons from different motor pools. Even though size ordered relationships between motor unit properties may be re-established in motor units which are appropriately reinnervated by their original motor axons, these relationships may be obscured by the data from misdirected motor units.

Thus, the purpose of the present study was to determine the pattern of innervation and recruitment of motor units in reinnervated human hand muscles after complete severance and resuture of the ulnar and median nerve at different levels.

\section{Methods}

\section{Clinical assessment}

Clinical assessments and experiments were all completed with the informed consent of the patients and according to the Declaration of Helsinki. All patients (two female, 11 male; 16-59 years) had accidentally severed the ulnar (six patients) or median (seven patients) nerve in the past 5 years. Surgical reports confirmed that the nerves had been completely sectioned and surgically repaired. All nerve sections were at wrist level except for two above-elbow median nerve sections.

The injured nerve and that of the control nerve in the other arm were each electrically stimulated at two levels to measure axonal conduction velocity. A bipolar needle electrode was inserted percutaneously into one or two reinnervated muscles in turn. The interference pattern was examined for the presence or absence of normal and polyphasic motor unit potentials. Muscle bulk and strength, together with general motor and sensory function were assessed clinically.

\section{Recruitment of reinnervated motor units}

Spike triggered averaging ${ }^{13}$ was used to record the twitch tension, contraction time, unrectified and rectified EMG of the reinnervated motor units in relation to the force at which they were recruited voluntarily.

EMG activity Motor unit potentials were recorded with a Disa bipolar needle electrode inserted percutaneously into the first dorsal interosseous or abductor digiti minimi muscle of the ulnar nerve patients and into the abductor pollicis brevis or opponens pollicis muscle of median nerve patients (fig 1). Surface EMG activity was recorded with two Beckman surface electrodes positioned on the belly of the muscle. Force measurements To measure the force of voluntary muscle contraction from the first dorsal interosseous and abductor digiti minimi muscles of ulnar nerve patients, a force transducer (Grass FT-03) was positioned against the proximal interphalangeal joint of the index and little finger respectively. For the median nerve patients, two strain gauges mounted at right angles (for details, see ${ }^{14}$ ) were positioned against the anterior and medial aspects of the thumb interphalangeal joint to record force from the abductor pollicis brevis and opponens pollicis muscles respectively.

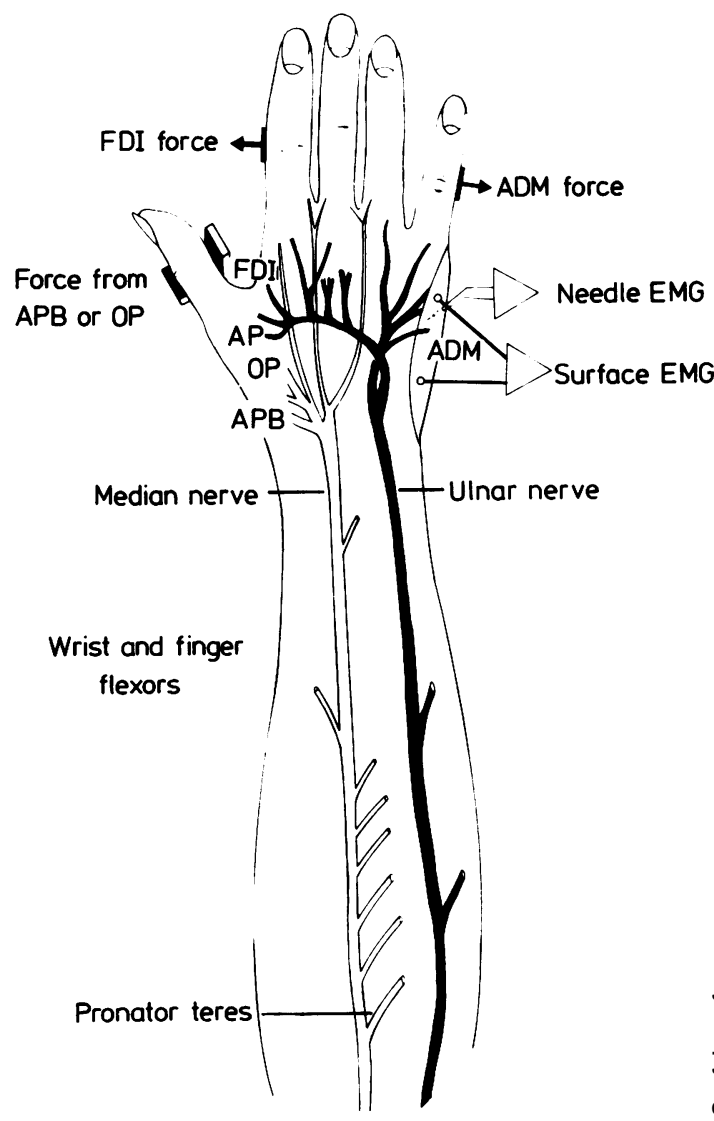

Fig 1 Schematic diagram of the path of the ulnar and median nerves helow the elbow joint. As each nerve travels down the forearm and into the hand, it innervates muscles with separate motor pools and different functions. The recording arrangement is only illustrated for the abductor digiti minimi $(A D M)$ muscle. How'ever, EMG from single motor units was recorded with bipolar needle electrodes from the FDI or the ADM muscle after ulnar nerve section and the abductor pollicis hrevis $A P B$ or opponens pollicis $(O P)$ muscle after median nerve section. Surface EMG was recorded from two electrodes positioned on the helly of the respective muscles. Force was recorded from the different muscles at the positions illustrated by the solid hars although only one or two transducers were used for each patient. The first dorsal interosseous (FDI), ADM, AP and other interossei and lumbrical muscles were all contracted in turn to determine the original motor pool of ulnarreinnervated motor units whereas the $A P B, O P$, pronator teres $(P T)$ and wrist and finger flexors were activated for motor pool identification of median-reinnervated motor units.

Spike triggered averaging Details of the spike triggered averaging technique have been described earlier. ${ }^{13}$ Briefly, each motor unit potential was accurately discriminated from the interference pattern (Bak Electronics Inc. window dis- 
criminator) and used to trigger a programme on a PDP 11/34 computer to average the force (filtered $0 \cdot 1-100 \mathrm{~Hz}$ ), unrectified and rectified EMG (filtered $10-10,000 \mathrm{~Hz}$ ) associated with the firing of the single motor unit. During each 500 sweep average, the subject was asked to recruit the motor unit and fire it slowly but repetitively at a force level near to the recruitment threshold (recorded continuously during a veraging with a digital voltmeter) to minimise the distortion in the twitch profile. ${ }^{1516}$ The averaged unrectified and rectified EMG was compared ${ }^{17}$ and indicated the absence of synchronised motor unit activity during averaging.

Identification of the original spinal pool of motor units After averaging the reinnervated motor unit parameters, the patient attempted to activate the same motor unit by voluntarily contracting different ulnar-innervated muscles in ulnar nerve sections (first dorsal interosseous, abductor digiti minimi, adductor pollicis, other interossei and lumbricals) or different median-innervated muscles in median nerve sections (abductor pollicis brevis, opponens pollicis, wrist and finger flexors, pronator teres; fig 1).

Normally innervated motor units were activated only by contraction of the muscle being recorded from. Reinnervated motor units that showed the same behaviour were considered to be appropriately innervated by motor axons from their original motor pool. Those reinnervated motor units that could be activated more easily by the contraction of other ulnar- or median-innervated muscles after the respective nerve sections, were considered to be reinnervated by motor axons from foreign motor pools. The original motor pool for these motor units was determined objectively from the muscle action which resulted in the recruitment of the motor unit in the recording muscle at the lowest level of force and rectified EMG. The patients confirmed subjectively that the motor unit was activated with the least effort by the contraction of that muscle.

Errors in this technique could arise when the respective muscles have closely synergistic functions. For example, in the abductor pollicis brevis muscle, motor units can be activated by abduction or opposition of the thumb. ${ }^{18}$ Following median nerve sections, these motor units were identified only as being of thenar muscle origin. Their actions could be separated clearly from those of the pronators and wrist and finger flexors. Similarly, the ulnar innervated first dorsal interosseous and adductor pollicis muscles can act as synergists. However, as first dorsal interosseous motor units do not co-operate in adduction of the thumb without flexion of the index finger ${ }^{14}$ motor units could be identified in these muscles by different muscle actions.

\section{Procedure}

In turn, different motor unit potentials were discriminated by having the subject exert various levels of voluntary force and/or by repositioning the needle electrode in the muscle. The mechanical properties of each motor unit were averaged and the motor unit pool identified as outlined above. One to three recording sessions of 1-2 hours were completed for each patient on different days.

\section{Analisis}

From the data from each patient, graphs of the twitch tension amplitude, contraction time and recruitment threshold were plotted on logarithmic coordinates. Data were also pooled and plotted similarly for ulnar nerve and median nerve sections. These data were compared to those obtained from subjects with normally innervated hand muscles.

Regression lines were fitted according to a least mean squares criterion. ${ }^{19}$ Lines were computed which minimised the mean square deviations in the $x$-direction ( $x$-line) and the $y$-direction ( $y$-line). The slope of the $y$-line is always the smaller of the two, and was used for statistical testing. This slope was taken to be significantly different from zero at the $5 \%$ level when its value was more than a factor of two greater than the standard error of the slope. Regression lines were only drawn when they satisfied this statistical criterion. The line which has a slope equal to the geometric mean (m-line) of the $x$-line and the $y$-line was also computed and was drawn in the figures of this paper. This line is appropriate when comparable errors of measurement occur in both the $\mathrm{x}$ - and $y$-values and is the line one would tend to fit by eye. ${ }^{14}$

The numbers of motor units from the different identified spinal motor pools in the reinnervated muscles were pooled separately for all ulnar and above-elbow median nerve section patients. A calculation was made of the contribution $(\%)$ of each muscle to the newly formed spinal motor pools after these different nerve sections.

\section{Results}

\section{Clinical assessment}

The injured nerves typically had lower conduction velocities than those recorded from the control nerves in the contralateral arm. The evoked compound muscle action potentials were smaller in amplitude and more dispersed than normal. In the reinnervated muscles, there was generally increased EMG activity with needle insertion and a reduced interference pattern composed of both normal and polyphasic motor unit potentials. Muscle strength and bulk were sometimes less than normal. Most patients had difficulty in isolating finger movements and described changes in their ability to complete fine motor skills such as fastening buttons and picking up small objects. Usually, tolerance to cold was reduced and tactile sensation was impaired.

In all patients, the motor and sensory deficits were specifically confined to the typical distribution of the nerve that had been injured. This suggested that there were no variations from the normal innervation of individual muscles although this does occur occasionally. ${ }^{6}$

\section{Innervation of reinnervated muscles}

The abductor digiti minimi, first dorsal interosseous and abductor pollicis brevis muscles were reinnervated appropriately by some of their original motor axons and inappropriately by motor axons that had previously innervated different muscles with different functions. Typical examples of the EMG patterns of reinnervated motor units are illustrated in fig 2 for the abductor digiti minimi muscle. The second 
(a) Normally directed motor unit

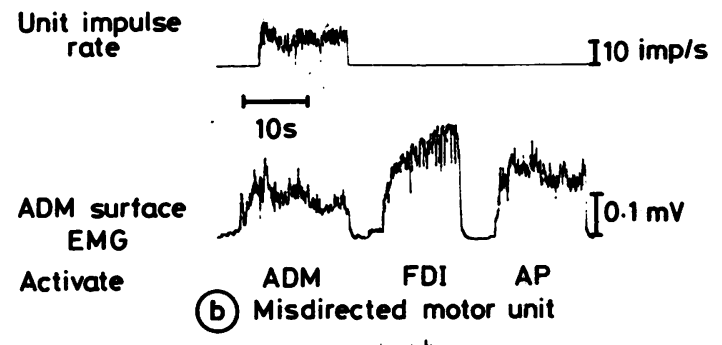

Unit impulse rate

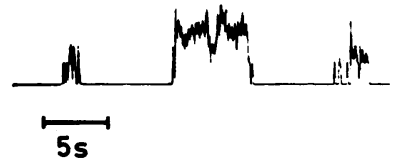

ADM surface EMG

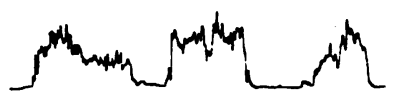

Fig 2 Unit impulse rate and rectified surface EMG activity of a normally directed (a) and misdirected (b) motor unit recorded in a reinnervated ADM muscle. EMG activity was recorded from the ADM muscle during contraction of either the ADM, FDI or AP muscles suggesting misdirection of many motor axones to that muscle. A normally directed unti (a) was activated only by the contraction of the ADM muscle. The motor axone had therefore appropriately reinnervated its original muscle. The misdirected motor unit (b), was activated most easily and at the lowest level of ADM rectified EMG by contraction of the FDI muscle so presumably belonged to the original FDI motor pool.

trace, in fig 2A, shows that surface EMG activity was always recorded in the abductor digiti minimi muscle when the abductor digiti minimi, first dorsal interosseous or adductor pollicis muscles were activated. As normal subjects can activate the abductor digiti minimi muscle independently of first dorsal inter- osseous or adductor pollicis, this suggests that many motor axons had been misdirected from other ulnar innervated muscles to the abductor digiti minimi muscle. Figure $2 \mathrm{~A}$ also shows an example of the typical EMG pattern of a normally directed motor unit. The motor unit fired during contraction of the abductor digiti minimi muscle but was silent during contraction of other ulnar reinnervated muscles such as the first dorsal interosseous or adductor pollicis. The motor axon had therefore appropriately reinnervated its original muscle. In comparison, misdirected motor axons from foreign motor pools formed motor units which could be activated by voluntary contraction of other muscles innervated by the same nerve (fig 2B). This motor unit was recruited at the lowest level of abductor digiti minimi rectified EMG by contraction of the first dorsal interosseous muscle. Higher levels of abductor digiti minimi rectified EMG had to be reached before the motor unit was recruited by the action of that muscle or the adductor pollicis muscle. Therefore, the motor unit was presumably in the spinal motor pool of the first dorsal interosseous muscle prior to the nerve section, but grew back to reinnervate muscle fibres in abductor digiti minimi.

The table shows the origin of the motor axons in the reinnervated muscles after ulnar and above-elbow median nerve section and resuture. After complete ulnar nerve section at wrist level, only $34 \%$ of the motor axons reinnervating the abductor digiti minimi muscle were from its original motor pool. The remaining motor axons had been misdirected from the original first dorsal interosseous $(31 \%)$, adductor pollicis $(18 \%)$ and other interossei and lumbrical $(17 \%)$ motor pools. Similarly, $39 \%$ of the motor axons reinnervating the first dorsal interosseous muscle were from the original first dorsal interosseous motor pool, whereas 11,22 and $28 \%$ were from the abductor digiti minimi, adductor pollicis and other interossei and lumbrical motor pools respectively. After median

Table Origin of motor axons in reinnervated muscles after complete ulnar or median nerve section and resuture

(A) Ulnar nerve sections at wrist level

Motor units activated most easily by contraction of:

\begin{tabular}{lllrr}
\cline { 2 - 4 } Muscle & $\begin{array}{l}\text { First dorsal } \\
\text { interosseous }\end{array}$ & $\begin{array}{l}\text { Abductor digiti } \\
\text { minimi }\end{array}$ & $\begin{array}{l}\text { Adductor } \\
\text { pollicis }\end{array}$ & Tothers \\
\hline Abductor digiti minimi & $15(31 \%)$ & $16(34 \%)$ & $9(18 \%)$ & $8(17 \%)$ \\
First dorsal interosseous & $22(39 \%)$ & $6(11 \%)$ & $12(22 \%)$ & $16(28 \%)$ \\
Total & $37(36 \%)$ & $22(21 \%)$ & $21(20 \%)$ & $24(23 \%)$
\end{tabular}

(B) Median nerve sections above the elbow

Motor units activated most easily by contraction of:

\begin{tabular}{lllll}
\cline { 2 - 4 } Muscle & Thenar & Pronator teres & Flexors & Total \\
\hline Abductor pollicis brevis & $20(48 \%)$ & $2(5 \%)$ & $14(33 \%)$ & $6(14 \%)$ \\
\hline
\end{tabular}

The numbers represent data from six ulnar and two above-elbow median nerve section patients. 


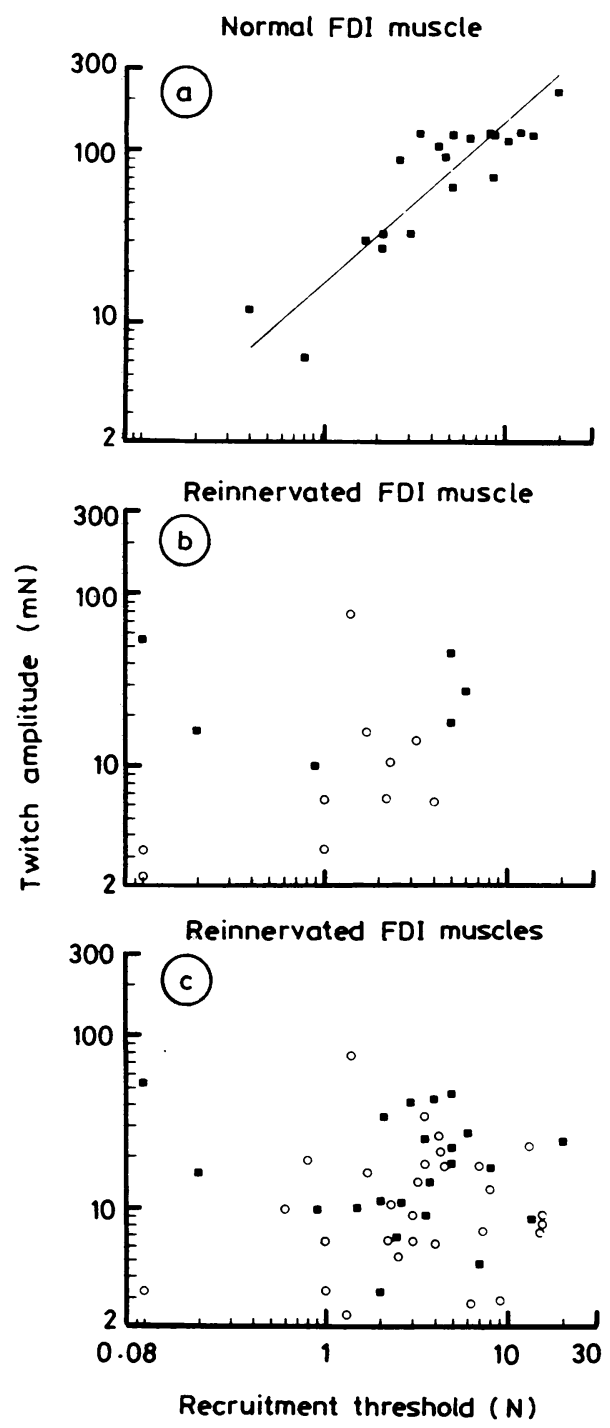

Fig 3 Motor unit twitch amplitude plotted against the threshold force of recruitment in a normally innervated FDI muscle (a), one (b) and four (c) reinnervated FDI muscles after ulnar nerve section and resuture at wrist level. Different symbols represent normally directed $(\square)$ and misdirected $(O)$ motor units in all graphs. Regression lines minimising the deviations in the $x$ and $y$ directions are draun in these and subsequent figs only when the relationships were significantly different from zero at the $5 \%$ level of confidence.

nerve section above the elbow, $48 \%$ of the reinnervated abductor pollicis brevis muscle motor pool was formed by original thenar (abductor pollicis brevis, opponens pollicis, flexor pollicis brevis) motor axons. Five, 33 and $14 \%$ of the motor axons were from the pronator teres, wrist and finger flexor or other motor pools respectively. Thus, these data clearly show that misdirection of motor axons occurs during reinnervation. However, this may not be an entirely random process because in both the first dorsal interosseous and abductor digiti minimi muscles, there was some tendency to record from a higher percentage of normally directed motor units than motor units from any one foreign motor pool.

\section{Size relationships in reinnervated muscles}

Figure 3 shows a typical example of the significant positive correlation found between the size of the motor unit (twitch amplitude) and the voluntary force at which the motor unit fired repetitively in a normally innervated first dorsal interosseous muscle (fig 3A). Following ulnar nerve section at wrist level, the range of reinnervated motor unit twitch tensions and recruitment thresholds was comparable with normal values. However, the correlation between these two variables was not. significant in an individual reinnervated muscle (fig $3 \mathrm{~B}$ ) or when data were pooled from several reinnervated muscles (fig $3 \mathrm{C}$ ). For normally directed motor units $(\square)$ in the first dorsal interosseous muscle, these correlations were positive but only significant in one muscle studied. Data recorded from reinnervated abductor digiti minimi muscles showed similar trends.

In a normally innervated abductor pollicis brevis muscle, significant positive correlations were also found between twitch tension amplitude and recruitment threshold (fig 4A). After median nerve section and resuture at the elbow, these relationships were not re-established in a reinnervated muscle (fig $4 \mathrm{C}$ ) or when the data from two muscles were pooled (fig 4E). In normally directed abductor pollicis brevis motor units $(\mathbb{a})$ these relationships were positive but not significant. In comparison, when the median nerve was sectioned and resutured at wrist level, the muscles were reinnervated by motor axons from motor pools with closely synergistic actions. Orderly relationships between recruitment threshold and twitch amplitude were re-established in a reinnervated muscle (fig 4B) or when data from several reinnervated muscles were pooled (fig 4D).

Figure 5 shows that there were significant negative correlations between motor unit contraction time and twitch amplitude in normally innervated first dorsal interosseous and abductor pollicis brevis muscles (fig 5A, 5B). Following ulnar or median nerve section and resuture, these correlations were weak (fig $5 \mathrm{C}$ ) or absent (fig 5D) in reinnervated muscles. The range of contraction times recorded in reinnervated muscles was similar to that recorded in the respective normal muscles. However, in all reinnervated muscles, the mean motor unit contraction time was faster than 


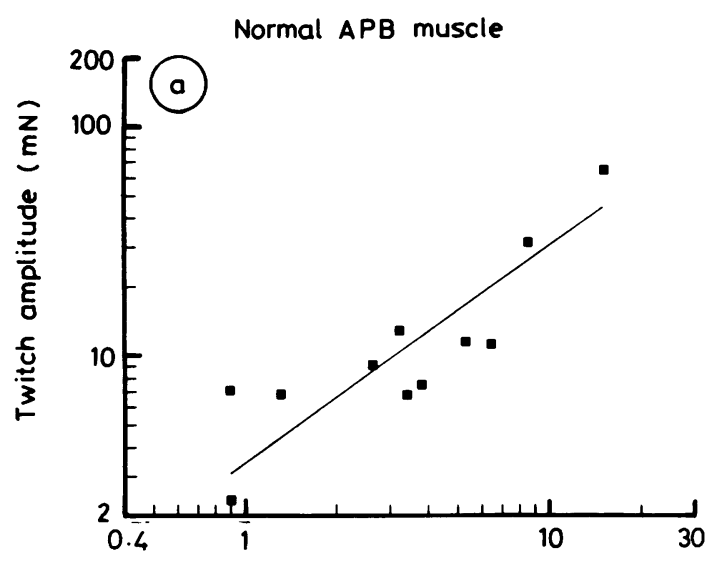

Reinnervated APB muscle
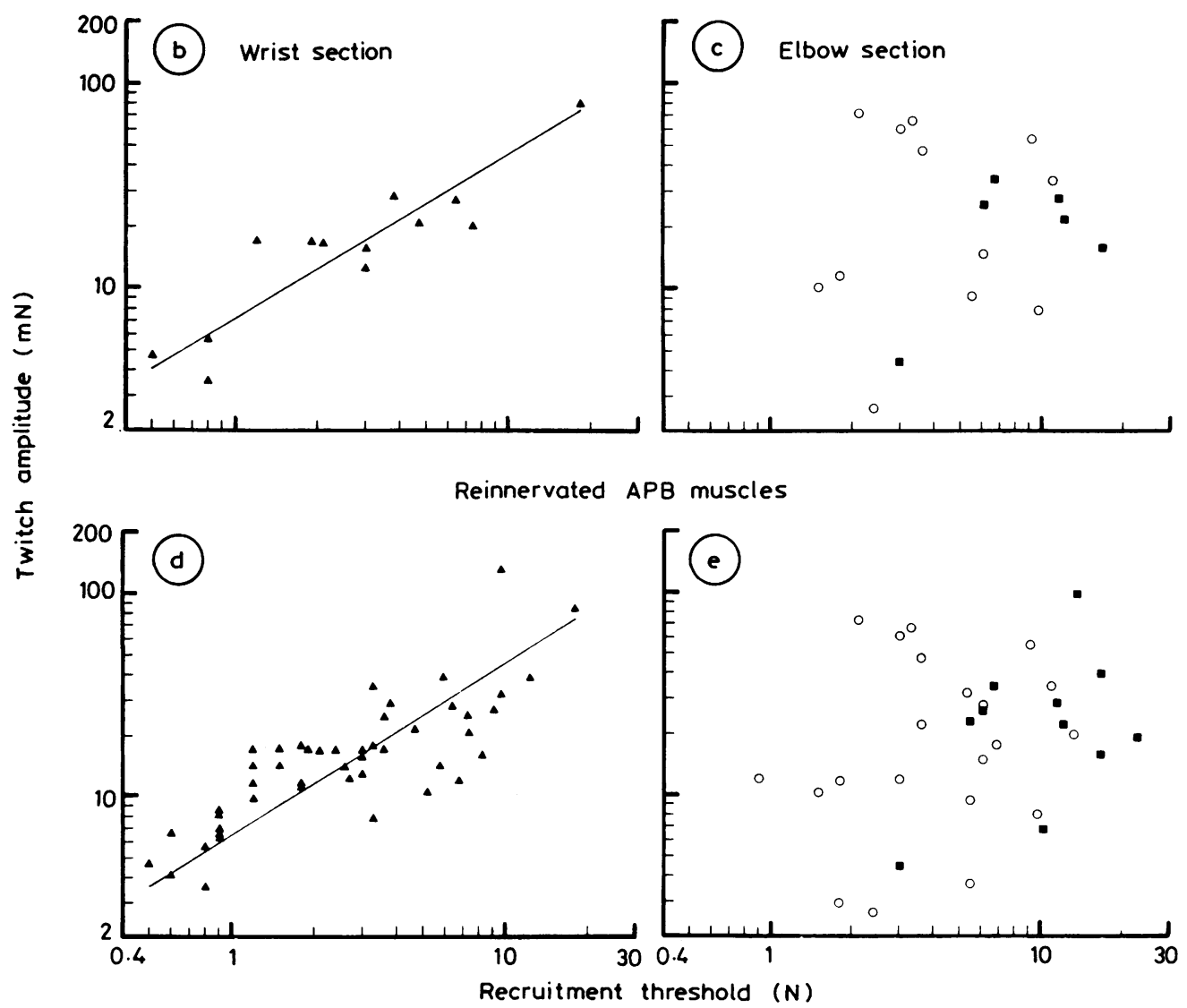

Fig 4 Motor unit twitch amplitude versus recruitment threshold in a normally innervated APB muscle (a). Data are plotted from one $(\mathrm{b}, \mathrm{c})$ and several $(\mathrm{d}, \mathrm{e})$ reinnervated $A P B$ muscles after median nerve section at the wrists and above the elbow respectively. Normally directed motor units $(\mathbf{\square})$ are represented by a different symbol to reinnervated motor units with motor axones of undetermined origin $(\Delta)$ and misdirected reinnervated motor units $(\bigcirc)$. 


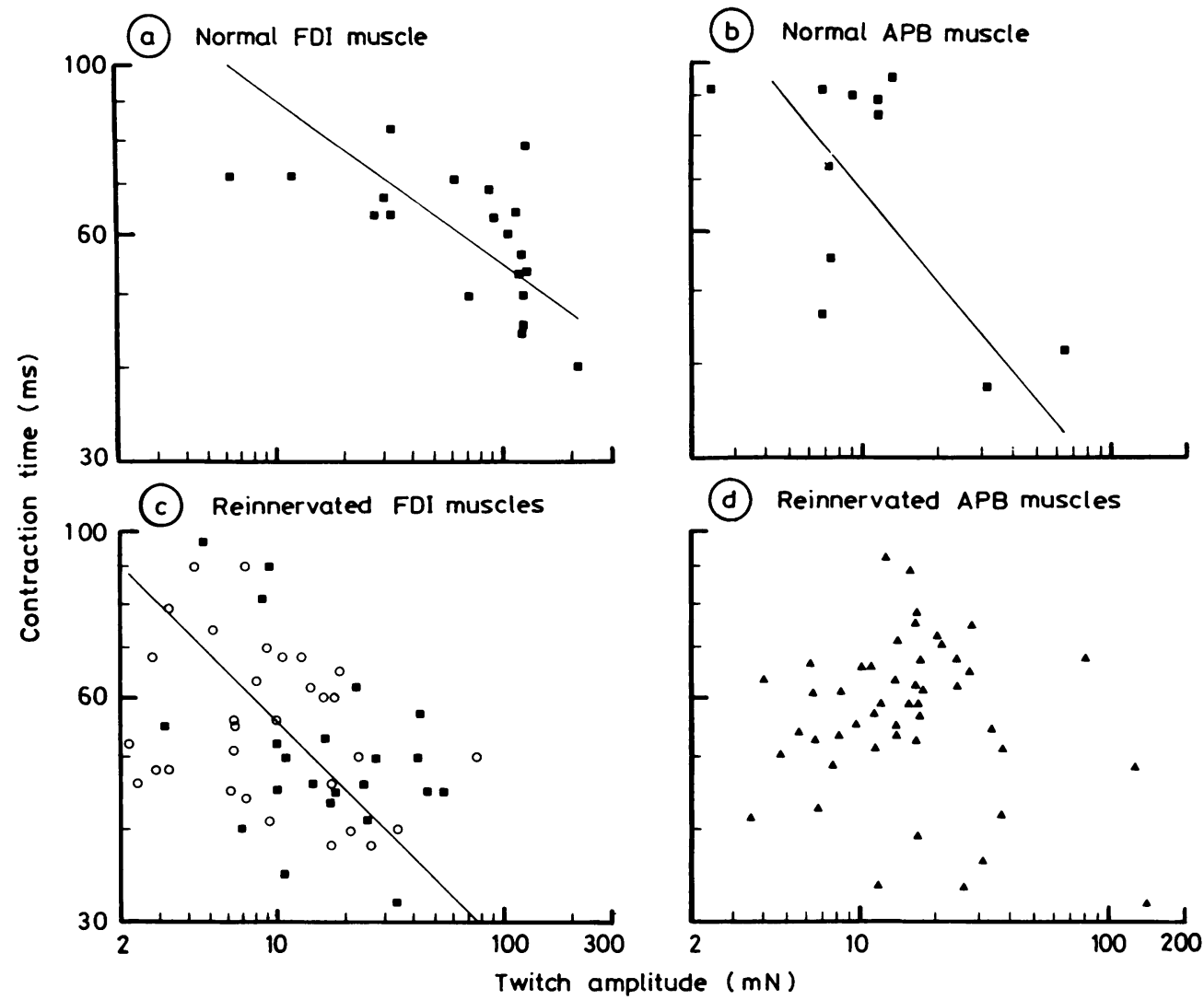

Fig 5 Motor unit contraction time plotted as a function of twitch amplitude in a normally innervated FDI (a) and $A P B$ (c) muscle. Data are plotted for reinnervated FDI (b) and APB (d) muscles after ulnar and above elhow-median nerve severance and repair respectively.

normal, but the differences were not statistically significant. As the patients sometimes found it difficult to activate a single motor unit at a low steady frequency, the erratic discharge pattern could have resulted in an underestimation of the contraction times for motor units.

\section{Discussion}

After complete ulnar or median nerve section and resuture in humans, regenerating motor axons were shown here either to reinnervate their original muscles or to be misdirected to foreign muscles. Only when motor axons reinnervate muscles with closely synergistic functions (median nerve at wrist), are orderly size relationships between motor unit properties re-established (fig 4B, 4D).

\section{Innervation of reinnervated muscles}

The poor motor coordination for fine movements observed clinically after peripheral nerve section and repair and the mass movements of facial muscles after facial nerve injury have sometimes been assumed to result from misdirection of regenerating motor axons. ${ }^{20-23}$ In the present study, this assumption was substantiated for peripheral nerve sections by identifying the original spinal motor pool of reinnervated motor units following ulnar and median nerve section. In the reinnervated first dorsal interosseous, abductor digiti minimi and abductor pollicis brevis muscles, two patterns of motor unit activity were recorded. Some motor units could be activated only by the action of the muscle in which they were recorded so they functioned normally. Presumably, the motor axons of these motor units reinnervated their original muscle. Other motor units could be activated best by contracting muscles innervated by the same nerve. These motor units were presumably formed by the motor axons being misdirected to reinnervate a foreign muscle. It was not tested to see if one axon innervated several muscles, but this is unlikely since axon sprouting usually occurs over very short distances. ${ }^{24}$ 
Similarly, the location of the cell body in the spinal cord was presumably unaltered. Following peripheral nerve section and resuture in animals, the location of the cell bodies remain intact and the motor axons exit from the appropriate ventral roots. ${ }^{25}$

Alternative explanations could be offered for the abnormal motor unit activity observed here. These include: (a) ephaptic transmission (b) alterations in the synaptic connections in higher brain centres (c) motor axons splitting to innervate different muscles. Impulses can be generated ectopically in chronically injured nerve fibres and possibly from hyperexcitability of the membrane. ${ }^{26}{ }^{27}$ Although this process may contribute to the motor unit activity recorded here, it seems unlikely that the impedance matching between the axons would be consistently appropriate to evoke transmission from local currents.

Monkeys ${ }^{28}$ and cats have been observed to perform well coordinated goal-directed movements after crossunion of large common nerves in the forearm. After deliberate cross-union of nerves, such re-education may result from compensation by the actions of synergistic muscles. In cats, the ability to perform coordinated voluntary movements may also result from sprouting of corticorubral neurons onto the proximal portion of the red nucleus. ${ }^{29}$ However in man, the red nucleus and the rubrospinal tract is less well developed. ${ }^{30}$ If synaptic alterations in these or other higher centres such as the corticospinal tract do occur in man after complete nerve section and repair, they do not appear to permit well coordinated finger movements. The patients in the present study reported difficulties in controlling their hand muscles during some functional tasks.

Examples of axon splitting have been reported in the extraocular and lumbrical muscles of the cat. ${ }^{31} 32$ This phenomenon is rare in human nerve regeneration. In some patients who have sectioned the ulnar $(2 / 18,11 \%)$ or facial $(1 / 50,4 \%)$ nerve, synchronous activity of some motor units was observed in different muscles reinnervated by the same nerve. ${ }^{20}$ Asynchronous motor unit discharges were much more common in all muscles examined, implying simple misdirection of the axon to a foreign muscle, as is proposed here.

From the present data, reinnervation of human hand muscles is clearly not specific. Similar findings have been reported in rat muscles when motor axons were provided with a choice of muscles to reinnervate. ${ }^{7}$ However, as more normally directed motor units than misdirected motor units from any one foreign pool were recorded in the abductor digiti minimi and first dorsal interosseous muscles after ulnar nerve section, this would suggest that reinnervation is not an entirely random process either. The abductor digiti minimi or first dorsal interosseous muscle can be activated by different actions to that of the adductor pollicis muscle so units activated by these muscle actions are unlikely to be mistaken with other motor pools. Alternatively, the tendency for more normally directed motor units to be recorded in these muscles may result from the careful rematching and resuture of the severed fascicles during surgery. This may provide the regenerating axons with a better opportunity to regrow along their original endoneurial tubes. Similarly, if the nerve section is quite close to the point of entry of the individual nerves into the muscles, there will be fewer branch points at which axons could be misdirected. The actual randomness of the reinnervation observed here could be substantiated by anatomical information on the numbers of motor axons in the ulnar nerve at the wrist and the respective muscle nerves, but this information is not currently available to our knowledge.

\section{Size relationships in reinnervated muscles}

Following ulnar and above-elbow median nerve section and resuture, the regenerating axons came from spinal pools with different functions. In the reinnervated first dorsal interosseous, abductor digiti minimi and abductor pollicis brevis muscles, motor unit recruitment was disordered. The ulnar nerve data confirm the previous findings of Milner-Brown et al. ${ }^{5}$ Furthermore, the data suggest that the lack of orderly motor unit recruitment by size after these complete nerve sections results from misdirection of motor axons from foreign motor pools.

In contrast, after median nerve section and resuture at the wrist, motor axons from synergistic motor pools reinnervated the denervated muscle fibres. Despite presumed misdirection of motor axons from foreign motor pools, orderly size relationships between motor unit properties were found in the abductor pollicis brevis muscles. Thus, orderly motor unit recruitment can be re-established after complete nerve section and resuture in humans, if the reinnervated muscles have closely synergistic functions. One factor favouring recovery after this injury may be the proximity of the section to the denervated muscle fibres. Near the level at which a muscle nerve leaves the main nerve trunk to innervate a muscle, the fibres of that individual nerve are sharply localised. ${ }^{6}$ With good fascicular repair after a clean cut, misdirection of axons may be reduced.

The relationships found between recruitment threshold and twitch amplitude in normally directed motor units did show some tendency for normal reorganisation and orderly recruitment. However, even though the size relationships for one muscle or one motor pool may be restored, these original pools were dispersed between different muscles after rein- 
nervation because of misdirection of regenerating motor axons. Thus, recruitment in any one muscle would depend on the recruitment of motor units from several pools with different functions. This was reflected by the difficulties the patients had in isolating individual finger movements. Typically, all muscles innervated by the same nerve would contract to some extent. These inappropriate movements may result from orderly recruitment of motor units by size within the original motor pools.

Data from animal studies support the proposal that misdirection of motor axons rather than a change in the activity of the motoneurons may explain a disordered recruitment of motor units and poor muscle control after complete nerve section and repair in humans. Full recovery of motoneuron properties and synaptic contacts onto axotomised motoneurons occurs following muscle innervation. ${ }^{33}$ When motoneurons reinnervate their former or antagonistic muscles, the physiological relationships between nerve and muscle properties also returned to normal. Thus, the tension and contractile speed of the muscle units were directly related to the amplitude of the extracellularly recorded action potential, as found in normally innervated muscles. ${ }^{1234}$ Self-reinnervated muscles function appropriately during locomotion. In comparison, cross-reinnervated cat muscles typically fired according to the pattern expected of the innervating motor pool during locomotion. ${ }^{35-37}$ Therefore, these data would tend to suggest that even though nerve and muscle unit properties are reestablished after nerve section and resuture, the motor units are recruited at a level of force that is appropriate within their original motor pool as is proposed here.

The impaired sensory function of the patients in the present study probably results from aberrant sensory reinnervation. Even though sensory axons reinnervate their end organs specifically, they are unlikely to reinnervate their original mechanoceptor. Misguided afferent fibres have been proposed to explain the perceptual mislocations of tactile stimuli following ulnar and median nerve section in humans ${ }^{38} 39$ and the abnormal response characteristics of muscle afferents after cross-reinnervation of the cat soleus muscle. ${ }^{40}$ Thus, even though the afferent responds to an appropriate mechanoceptor, the localisation of the sensory stimulus may be interpreted incorrectly. This mismatching of sensory connections may be distorted further by misdirection of the motor axons to foreign muscles.

In conclusion, after complete ulnar or median nerve section and resuture in humans, the reinnervation of hand muscles was shown to be abnormal physiologically. Motor axons reinnervated their original or foreign muscles. Even so, the reinnervated muscles served some useful function during power or gripping movements. Finely coordinated movement sequences were impaired, in part due to misdirection of motor axons and in part to impaired tactile sensation. Thus, the data in this study not only emphasise the need for largely preserving the pattern of nerve and muscle connections for size ordered motor unit recruitment, but also show the importance of this pattern of recruitment for control of fine movements.

This research was supported by grants from the Medical Research Council of Canada and the Muscular Dystrophy Association of Canada. CKT was a Commonwealth Scholar.

\section{References}

1 Clough JFM, Kernell D, Phillips CG. The distribution of monosynaptic excitation from the pyramidal tract and from primary spindle afferents to motoneurons of the baboon's hand and forearm. I Physiol (Lond) 1968;198:145-66.

2 Lawrence DG, Kuypers HGJM. Pyramidal and nonpyramidal pathways in monkeys: anatomical and functional correlation. Science 1965;148:973-5.

3 Henneman E, Somjen G, Carpenter DO. Functional significance of cell size in spinal motoneurons. J Neurophysiol 1965;28:560-80.

4 Milner-Brown HS, Stein RB, Yemm R. The orderly recruitment of human motor units during voluntary isometric contractions. J Physiol (Lond) 1973;230: 359-70.

5 Milner-Brown HS, Stein RB, Lee RG. Pattern of recruiting human motor units in neuropathies and motorneuron disease. J Neurol Neurosurg Psychiatry 1974;37:665-9.

6 Sunderland S. Nerve and Nerve Injuries. London: Livingstone, 1978.

7 Gillespie MJ, Gordon T, Murphy PR. Reinnervation of the lateral gastrocnemius and soleus muscles in the rat by their common nerve. $J$ Physiol (Lond) 1986;372: 485-500.

8 Dubowitz V. Pathology of experimentally re-innervated skeletal muscle. J Neurol Neurosurg Psychiatry 1967;30:99-110.

9 Karpati G, Engel WK. "Type-grouping" in skeletal muscles after experimental reinnervation. Neurology 1968;18:447-55.

10 Ariano MA, Armstrong RB, Edgerton VR. Hindlimb muscle fibre populations of five mammals. J Histochem Cytochem 1973;21:51-5.

11 Kugelberg E, Edström L, Abbruzzese M. Mapping of motor units in experimentally reinnervated rat muscle. Interpretation of histochemical and atrophic fibre patterns in neurogenic lesions. $J$ Neurol Neurosurg Psychiatry 1970;33:319-29.

12 Gordon T, Stein RB. Reorganization of motor-unit properties in reinnervated muscles of the cat. $J$ Neurophysiol 1982;48:1175-90.

13 Stein RB, French AS, Mannard D, Yemm R. New 
methods for analyzing motor function in man and animals. Brain Res 1972;40:187-92.

14 Thomas CK, Ross BH, Stein RB. Motor-unit recruitment in human first dorsal interosseous muscle for static contractions in three different directions. J Neurophysiol 1986;55:1017-29.

15 Andreassen S, Bar-On E. Estimation of motor unit twitches. IEEE Trans Biomed Eng 1983;30:742-8.

16 Calancie B, Bawa P. Limitations of the spike triggered averaging technique. Muscle Nerve 1986;9:78-84.

17 Milner-Brown HS, Stein RB, Yemm R. The contractile properties of human motor units during voluntary isometric contraction. J Physiol (Lond) 1973;228: 280-306.

18 Ross BH, Thomas CK, Calancie B. Human motor unit recruitment during static contractions in different directions and during dynamic unrestrained movement. Neurosci Abs 1985;11:328.

19 Sokolnikoff IS, Redheffer RM. In: Mathematics of Physics and Modern Engineering. New York: McGraw-Hill, 1958.

20 Esslen E. Electromyographic findings on two types of misdirection of regenerating axons. Electroencephaeogr Clin Neurophysiol 1960;12:738-41.

21 Ford FR, Woodhall B. Phenomena due to misdirection of regenerating fibres of cranial, spinal and autonomic nerves. Arch Surg 1938;36:480-96.

22 Frueh BR. Associated facial contractures after seventh nerve palsy mimicking jaw-winking. Ophthalmology 1983;90:1105-9.

23 Kimura J, Rodnitzky RL, Okawara S-H. Electrophysiologic analysis of aberrant regeneration after facial nerve paralysis. Neurology 1975;25:989-93.

24 Slack JR, Pockett S. Terminal sprouting of motoneurones is a local response to a local stimulus. Brain Res 1981;217:368-74.

25 Brushank TM, Mesulam MM. Alterations in connections between muscle and anterior horn motoneurons after peripheral nerve repair. Science 1980;208:603-5.

$26 \mathrm{Krnjevic} \mathrm{K}$. Ephaptic interactions: a significant mode of communications in the brain. News in Physiological Sciences 1986;1:28-9.

27 Rasminsky M. Ectopic impulse generation in pathological nerve fibres. Trends in Neurosciences 1983;6:368-70.

28 Brinkman C, Porter R, Norman J. Plasticity of motor behavior in monkeys with crossed forelimb nerves. Science 1983;220:438-40.
29 Tsukahara N, Fujito Y, Oda Y, Maeda J. Formation of functional synapses in the adult cat red nucleus from the cerebellum following cross-innervation of forelimb flexor and extensor nerves. Exp Brain Res 1982; 45: $1-12$.

30 Carpenter MB. Anatomy of the corpus striatum and brain stem integrating systems. In: Handbook of Physiololgy. The Nervous System. Bethesda MD: Am Physiol Soc Sect 1. Vol. 2. 1981:947-96.

31 Crandell WF, Goldberg SJ, Wilson JS, McClung JR. Muscle units divided among retractor bulbi muscle slips and between the lateral rectus and retractor bulbi muscles in cat. Exp Neurol 1981;71:251-60.

32 Emonet-Denand F, Laporte Y, Proske U. Contraction of muscle fibres in two adjacent muscles innervated by branches of the same motor axon. $J$ Neurophysiol 1971;34:132-8.

33 Sumner BEH, Sutherland FI. Quantitative electron microscopy on the injured hypoglossal nucleus in the rat. $J$ Neurocytol 1973;2:315-28.

34 Gordon T, Stein RB, Thomas CK. Organization of motor units following cross-reinnervation of antagonistic muscles in the cat hindlimb. $J$ Physiol (Lond) 1986;374:442-56.

35 Gordon T, Stein RB, Thomas CK. Innervation and function of hind-limb muscles in the cat after cross-union of the tibial and peroneal nerves. $J$ Physiol (Lond) 1986;374:429-41.

36 Luff AR, Webb SN. Electromyographic activity in the cross-reinnervated soleus muscle of unrestrained cats J Physiol (Lond) 1985;365:13-28.

37 O'Donovan MJ, Pinter MJ, Dum RP, Burke RE Kinesiological studies of self- and cross-reinnervated FDL and soleus muscles in freely moving cats. J Neurophysiol 1985;54:852-66.

38 Hallin RG, Wiesenfeld Z, Lindblom U. Neurophysiological studies on patients with sutured median nerves: faulty sensory localization after nerve regeneration and its physiological correlates. Exp Neurol 1981;73:90-106

39 Mackel R, Kunesch E, Waldhör F, Struppler A. Reinnervation of mechanoreceptors in the human glabrous skin following peripheral nerve repair. Brain Res 1983;268:49-65.

40 Gregory JE, Luff AR, Proske U. Muscle receptors in the cross-reinnervated soleus muscle of the cat. $J$ Physiol (Lond) 1982;331:367-83. 